Original article

\title{
Gender difference in oral health perception and practices among Medical House Officers
}

\author{
Clement C. Azodo and Barnabas Unamatokpa
}

Department of Periodontics, University of Benin Teaching Hospital, Benin City, Nigeria

Received 1 July 2012, Accepted 10 July 2012

(C) 2012, Azodo C.C., Unamatokpa B.

(C) 2012, Russian Open Medical Journal

\begin{abstract}
Background: Understanding the effect of gender on oral health would facilitate the development of successful attitude and behavior modification approach towards sustainable oral health. Aim: To assess the gender difference in the oral health perception and practices among medical house officers in Benin City, Nigeria. Materials and Methods: This questionnaire-based cross-sectional study was conducted among doctors undergoing 12-months mandatory housemanship in tertiary and secondary hospitals in Benin City, Nigeria. The questionnaire assessed information on demography, perceived oral health, oral self-care, dental visit and oral disease preventive knowledge. Results: Of the 105 questionnaires distributed, 97 questionnaires were filled and returned giving a response rate of $92.4 \%$. Females in comparison to males significantly gave good attention to their oral health, use medium strength toothbrush, brushed teeth more than once-daily, visited dentist and chose toothpaste following dentist recommendations. There was no significant gender difference in self-reported dental problem, perceived oral health, preventive knowledge in relation to dental caries and gingival bleeding, the reasons for tooth brushing and renewal of toothbrush. Conclusion: Gender played a role in the perception of general health relative oral health, dental visit, daily tooth brushing frequency and choice of toothbrush and toothpaste for oral self-care. The development of oral health attitude and behavior modification approaches towards sustainable oral health among the studied group should reflect these differences.
\end{abstract}

Keywords: gender difference, perceived oral health, oral health practices, house officers.

Cite as Azodo CC, Unamatokpa B. Gender difference in oral health perception and practices among Medical House Officers. Russian Open Medical Journal 2012; 1: 0208 .

Correspondence to Dr Clement C. Azodo. Address: Department of Periodontics New Dental Complex, University of Benin Teaching Hospital, P.M.B. 1111 Ugbowo, Benin City, Edo State, Nigeria 300001. Phone: +2348034051699. Email: clementazodo@yahoo.com

\section{Introduction}

Oral diseases constitute public health problem in developing countries due to their high prevalence, economic consequences and negative impact on the quality of life of affected individual. Oral diseases adversely affect concentration, interpersonal relationship and productivity due to the intricate relationship between oral health and general health. Prevention of oral disease can be achieved by optimal oral health practices which include tooth brushing, flossing, dental visits and proper dietary practices.These preventive oral health practices are influenced by socioeconomic status, with individuals of high socioeconomic status in which doctors belong, exhibiting optimal preventive oral health practices. The adoption of optimal oral health practices among healthcare workers especially doctors is expected to positively influence the public because of their dependence on them for proper health knowledge, attitude and practices. The high dependence on doctors for oral health information due to ignorance among individuals, shortage and inequitable distribution of oral healthcare manpower in Nigeria has been documented. It is therefore important to assess the oral health perception and practices among doctors to ascertain the baseline information that will help in the development of programmes that will fill the noted gaps. The assessment of oral health perception and practices designed with gender inclination will yield additional information which may result in success oriented programmes.
Several studies on oral health knowledge, attitude and practices among health workers were conducted predominantly among dental health professionals neglecting non dental health professionals $[1,2]$. Among the few studies whose participants were non dental health professionals, inadequate oral health knowledge and suboptimal practices were reported and also left out the influence of gender on the oral health.

Although widespread publications on gender differences in general heath exist in the literature but those restricted to oral health are scanty [3-8]. The studies available in the literature on gender differences in relation to oral health were conducted in the Asia (Japan) [9-11], Europe (Sweden) [12, 13], and Middle East (Jordan) [1], Kuwait [14] and Palestine [2] and North Africa (Libya) [15]. The findings from theses studies $[1,2,8-15]$ consistently revealed that females are more informed about tooth brushing, have more interest in oral health and perceive their own oral health to be good to a higher degree than male. They also exhibit more positive dental health attitude and better oral health behaviour (tooth brushing frequency; using dental floss; regular dental visits) than their male counterparts. The variations in geographic location, cultural and religious belief in the studied groups in different countries with Nigeria suggest the need for such a study to compare with these documented evidences in the literature. The aim of the study was to assess the gender 
difference in the oral health perception and practices among medical house officers in Benin City, Nigeria.

\section{Material and Methods}

This cross sectional study of doctors undergoing 12 months mandatory housemanship in University of Benin Teaching Hospital and Central Hospital Benin City was undertaken in last quarter of 2010. Using the formula for calculating sample size for crosssectional study, the minimum sample size $=\frac{Z=P q}{d z}=82$.

$Z=1.96$ which $95 \%$ confidence.

$p=$ proportion of medical doctors that have good knowledge of conditions referred to appropriate specialty. $P=5.6 \%[16]$ and $q$ $=1-p$.

The estimated sample size with $10 \%$ added to compensate for non-response made a total of 91 respondents, the minimum sample size to provide acceptable precision. However, a total of 97 respondents participated in the study of 105 questionnaires distributed. The questionnaire assessed information on demography, oral self-care and dental visit. Information relating to toothbrush and tooth brushing were also assessed as thus: the primary and secondary reasons for tooth brushing, frequency of tooth brushing, texture of toothbrush and timing of renewal of tooth brush. The knowledge in relation to the prevention of dental caries and gingival bleeding were assessed and the responses were categorized as no knowledge, incomplete knowledge and complete knowledge. Perceived oral health status was assessed using a single question global oral health rating with responses as excellent, good, fair and poor. The perceived health status was categorized as poor/fair and excellent/good for the purpose of analysis.

The questionnaires were anonymous in nature and contained no identifiers. Informed consent was obtained from participants after informing them of the objective of the study. Participation in the study was voluntary and no incentive was offered. The data analysis was done in a personal computer using Statistical Package for the Social Sciences (SPSS) version 17.0. The gender difference in oral health knowledge, attitude and practices among the participants was assessed using Chi-square statistics and Fischer's exact. $\mathrm{P}<0.05$ was considered to be statistically significant.

\section{Results}

Out of 105 questionnaires distributed, 97 questionnaires were returned filled giving a response rate of $92.4 \%$. The female respondents were generally younger than the male respondents (Table 1). Females in comparison to the males significantly had more dental visits and gave good attention to their oral health (Table 2). Females in comparison to the males significantly used medium strength toothbrush, brushed their teeth more once-daily (Table 3) and choose toothpaste following dentist recommendations (Table 4). There was no significant gender difference in the reported primary and secondary reasons for tooth brushing and renewal of tooth brush (Table 5) and knowledge in relation to the prevention of dental caries and gingival bleeding (Table 6).

Table 1. Gender differences in the age distribution of the respondents

\begin{tabular}{lcccc}
\hline \multirow{2}{*}{ Age, years } & \multirow{2}{*}{ Total, $\mathrm{n}(\%)$} & \multicolumn{2}{c}{ Gender } & \multirow{2}{*}{ P-Value } \\
& & Male, $\mathrm{n}(\%)$ & Female, $\mathrm{n}(\%)$ & \\
\hline$<25$ & $9(9.3)$ & $1(1.8)$ & $8(20.0)$ & 0.010 \\
$25-27$ & $46(47.4)$ & $26(45.6)$ & $20(50.0)$ & \\
$28-30$ & $33(34.0)$ & $24(42.1)$ & $9(22.5)$ & \\
$>30$ & $9(9.3)$ & $6(10.5)$ & $3(7.5)$ & \\
\hline Total & $97(100.0)$ & $57(100.0)$ & $40(100.0)$ & \\
\hline
\end{tabular}

Table 2. Gender differences in oral health perception, dental visit and oral health problems

\begin{tabular}{|c|c|c|c|c|}
\hline \multirow{2}{*}{ Characteristics } & \multirow{2}{*}{ Total, n (\%) } & \multicolumn{2}{|c|}{ Gender } & \multirow{2}{*}{ P-Value } \\
\hline & & Male, n (\%) & Female, n (\%) & \\
\hline \multicolumn{5}{|c|}{ Perceived oral health status } \\
\hline Poor/fair & $34(35.1)$ & $23(40.4)$ & $11(27.5)$ & 0.192 \\
\hline Good/excellent & $63(64.9)$ & $34(59.6)$ & $29(72.5)$ & \\
\hline \multicolumn{5}{|c|}{ Equal care for oral and general health } \\
\hline Yes & $78(80.4)$ & $42(73.7)$ & $36(90.0)$ & 0.047 \\
\hline No & $19(19.6)$ & $15(26.3)$ & $4(10.0)$ & \\
\hline \multicolumn{5}{|l|}{ Dental visit } \\
\hline Yes & $37(38.1)$ & $16(28.1)$ & $21(52.5)$ & 0.015 \\
\hline No & $60(61.9)$ & $41(71.9)$ & $19(47.5)$ & \\
\hline \multicolumn{5}{|c|}{ Oral health problem } \\
\hline Yes & $24(24.7)$ & $18(31.6)$ & $6(15.0)$ & 0.063 \\
\hline No & $73(75.3)$ & $39(68.4)$ & $34(85.0)$ & \\
\hline Total & $97(100.0)$ & $57(100.0)$ & $40(100.0)$ & \\
\hline
\end{tabular}

Table 3. Gender differences in toothbrush and tooth brushing behaviours

\begin{tabular}{|c|c|c|c|c|}
\hline \multirow{2}{*}{ Characteristics } & \multirow{2}{*}{ Total, n (\%) } & \multicolumn{2}{|c|}{ Gender } & \multirow{2}{*}{ P-Value } \\
\hline & & Male, n (\%) & Female, $\mathrm{n}(\%)$ & \\
\hline \multicolumn{5}{|c|}{ Daily brushing frequency } \\
\hline \multirow{2}{*}{$\begin{array}{l}\text { Once daily } \\
\text { More than } \\
\text { once daily }\end{array}$} & $31(32.0)$ & $27(47.4)$ & $4(10.0)$ & \multirow[t]{2}{*}{$<0.001$} \\
\hline & $66(68.0)$ & $30(52.6)$ & $36(90.0)$ & \\
\hline \multicolumn{5}{|c|}{ Strength of toothbrush } \\
\hline Soft & $11(11.3)$ & $7(12.3)$ & $4(10.0)$ & \multirow[t]{3}{*}{$0.027^{F}$} \\
\hline Medium & $64(66.0)$ & $32(56.1)$ & $32(80.0)$ & \\
\hline Hard & $22(22.7)$ & $18(31.6)$ & $4(10.0)$ & \\
\hline \multicolumn{5}{|c|}{ Toothbrush renewal } \\
\hline Every 3 months & $39(40.2)$ & $27(47.4)$ & $12(30.0)$ & \multirow[t]{5}{*}{$0.320^{F}$} \\
\hline Frayed bristles & $41(42.3)$ & $19(33.3)$ & $22(55.0)$ & \\
\hline Missing & $6(6.2)$ & $4(7.0)$ & $2(5.0)$ & \\
\hline Broken & $6(6.2)$ & $4(7.0)$ & $2(5.0)$ & \\
\hline Others & $5(5.2)$ & $3(5.3)$ & $2(5.0)$ & \\
\hline Total & $97(100.0)$ & $57(100.0)$ & $40(100.0)$ & \\
\hline
\end{tabular}

Table 4. Gender differences in primary and secondary reasons for tooth brushing

\begin{tabular}{|c|c|c|c|c|c|c|}
\hline \multirow{2}{*}{ Reasons } & \multicolumn{2}{|c|}{ Gender } & \multirow{2}{*}{ Total, n (\%) } & \multicolumn{2}{|c|}{ Gender } & \multirow{2}{*}{ Total, n (\%) } \\
\hline & Male, n (\%) & Female, n (\%) & & Male, n (\%) & Female, n (\%) & \\
\hline & Primary & & & Secondary & & \\
\hline Clean bright teeth & $13(22.8)$ & $17(42.5)$ & $30(30.9)$ & $22(38.6)$ & $20(50.0)$ & $42(43.3)$ \\
\hline Preventing dental caries/ periodontal diseases & $26(45.6)$ & $18(45.0)$ & $44(45.4)$ & $9(15.8)$ & $7(17.5)$ & $16(16.5)$ \\
\hline Preventing halitosis & $15(26.3)$ & $5(12.5)$ & $20(20.6)$ & $20(35.1)$ & $13(32.5)$ & $33(34.0)$ \\
\hline Preventing tooth colour change & $3(5.3)$ & $0(0.0)$ & $3(3.1)$ & $3(5.3)$ & $0(0.0)$ & $3(3.1)$ \\
\hline Preventing oral ulcer & - & $\ldots$ & - & $3(5.3)$ & $0(0.0)$ & $3(3.1)$ \\
\hline Total & $57(100.0)$ & $40(100.0)$ & $97(100.0)$ & $57(100.0)$ & $40(100.0)$ & $97(100.0)$ \\
\hline
\end{tabular}


Stomatology

Table 5. Gender differences in the factors influencing the choice of toothpaste

\begin{tabular}{|c|c|c|c|c|}
\hline \multirow{2}{*}{ Factors } & \multirow{2}{*}{ Total, n (\%) } & \multicolumn{2}{|c|}{ Gender } & \multirow{2}{*}{ P-Value } \\
\hline & & Male, n (\%) & Female, n (\%) & \\
\hline Dentist recommendation & $63(64.9)$ & $29(50.9)$ & $34(85.0)$ & $0.022^{F}$ \\
\hline Media advertisement & $13(13.4)$ & $11(19.3)$ & $2(5.0)$ & \\
\hline Flavour of toothpaste & $7(7.2)$ & $6(10.5)$ & $1(2.5)$ & \\
\hline Stop mouth odour & $4(4.1)$ & $4(7.0)$ & $0(0.0)$ & \\
\hline Parent use & $3(3.1)$ & $2(3.5)$ & $1(2.5)$ & \\
\hline Whitening & $3(3.1)$ & $2(3.5)$ & $1(2.5)$ & \\
\hline Others & $4(4.1)$ & $3(5.3)$ & $1(2.5)$ & \\
\hline Total & $97(100.0)$ & $57(100.0)$ & $40(100.0)$ & \\
\hline
\end{tabular}

F-Fisher's P

Table 6. Gender differences in knowledge about the prevention of dental caries and gingival bleeding

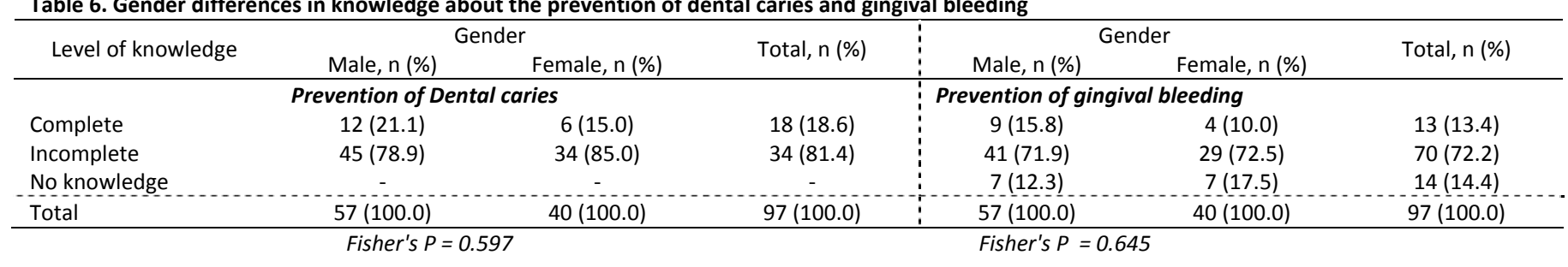

\section{Discussion}

In this study, female participants significantly gave good attention to their oral health, used medium strength toothbrush, brush their teeth more than once-daily, and choose toothpaste following dentist recommendations than male participants. The attachment of higher importance to oral health like general health and the significant dental visit among females explained the significant use of proper toothbrush strength, tooth brushing frequency and the choice of toothpaste following dentist recommendations. This aligned with the report of a study among dental students which showed that female students believed in the necessity of using toothpaste during brushing and brushed their teeth more frequently than the male students [1]. This is in tandem with findings of studies among Palestinian undergraduate dental students [2], Japanese dental patients [10], and Kuwait Health Sciences College students [14] where female students had more positive dental health attitudes and behaviours (regular dental visits, professional tooth brushing education and tooth brushing behaviour and frequency and dental floss use) in comparison with their male counterpart. The fact that female students were exposed to more oral health information, had stronger oral beliefs and performed preventive behaviours more frequently than did their male counterparts is a possible explanation [17]. It also explained the significant gender difference in the presence of calculus around the teeth in the mouth [18]. The gender gap in oral health may have arisen from genetic, hormonal, and cultural influences [19].

There was no significant gender difference in the self-reported dental problem, dental visit, perceived oral health status, knowledge in relation to the prevention of dental caries and gingival bleeding, the primary and secondary reasons for tooth brushing and renewal of tooth brush. The non-significantly higher perception of oral health as good among males than females contrasted with findings of studies in Skaraborg County, Sweden where girls perceived their own oral health to be good to a higher degree than boys $[12,13]$. The influence of age on male and female health behaviour may be responsible for the contrasting finding as this study group were young adults while the compared studies were adolescents.

\section{Conclision}

Gender played a role in the perception of general health relative oral health, dental visit, daily tooth brushing frequency and choice of toothbrush and toothpaste for oral self-care. The development of oral health attitude and behavior modification approaches towards sustainable oral health among the studied group should reflect these differences.

\section{Conflict of interest}

The authors declare that they have no conflict of interest.

\section{Acknowledgement}

The abstract of this article was presented at 5th Annual Scientific Conference of the Faculty of Dental Sciences, College of Medicine, University of Lagos on 6th July, 2011.

\section{Reference}

1. Al-Omari QD, Hamasha AA. Gender-specific oral health attitudes and behavior among dental students in Jordan. J Contemp Dent Pract 2005; 6: 107-114 (PMID: 15719082).

2. Kateeb E. Gender-specific oral health attitudes and behaviour among dental students in Palestine. East Mediterr Health J 2010; 16: 329-333 (PMID: 20795450).

3. Verbrugge LM. Sex differentials in health. Public Health Rep 1982; 97 417-437 (PMCID: 1424355).

4. Verbrugge LM, Wingard DL. Sex differentials in health and mortality. Women Health 1987; 12: 103-145 (PMID: 3424846) (doi: 10.1300/J013v12n02 07).

5. Macintyre S, Ford G, Hunt K. Do women 'over-report' morbidity? Men's and women's responses to structured prompting on a standard question on long standing illness. Soc Sci Med 1999; 48: 89-98 (PMID: 10048840) (doi: 10.1016/S0277-9536(98)00292-5).

6. Lahelma E, Martikainen $P$, Rahkonen $O$, Silventoinen K. Gender differences in ill health in Finland: patterns, magnitude and change. Soc Sci Med 1999; 48: 7-19 (PMID: 10048834) (doi: 10.1016/S02779536(98)00285-8).

7. Ladwig KH, Marten-Mittag B, Formanek B, Dammann G. Gender differences of symptom reporting and medical health care utilization in the German population. Eur J Epidemiol 2000; 16: 511-518 (PMID: 11049093) (doi: 10.1023/A:1007629920752).

8. Redondo-Sendino A, Guallar-Castillón $P$, Banegas JR, RodríguezArtalejo F. Gender differences in the utilization of health-care services among the older adult population of Spain. BMC Public Health 2006; 6: 155 (PMCID: 1525176) (doi: 10.1186/1471-2458-6-155). 
9. Fukai K, Takaesu Y, Maki Y. Gender differences in oral health behavior and general health habits in an adult population. Bull Tokyo Dent Coll 1999; 40: 187-193 (PMID: 11933308) (doi: 10.5580/20ce).

10. Tada A, Hanada N. Sexual differences in oral health behaviour and factors associated with oral health behaviour in Japanese young adults. Public Health 2004; 118: 104-109 (PMID: 15037039) (doi: 10.1016/i.puhe.2003.05.007).

11. Furuta M, Ekuni D, Irie K, Azuma T, Tomofuji T, Ogura T, Morita M. Sex differences in gingivitis relate to interaction of oral health behaviors in young people. J Periodontol 2011; 82: 558-565 (PMID: 20936916) (doi: 10.1902/jop.2010.100444).

12. Ostberg AL, Halling A, Lindblad U. Gender differences in knowledge, attitude, behavior and perceived oral health among adolescents. Acto Odontol Scand 1999; 57: 231-236 (doi: 10.1080/000163599428832) (PMID: 10540935).

13. Ostberg AL, Halling A, Lindblad U. A gender perspective of selfperceived oral health in adolescents: associations with attitudes and behaviours. Community Dent Health 2001; 18: 110-116 (PMID: 11461058).

14. Al-Ansari JM, Honkala S. Gender differences in oral health knowledge and behavior of the health science college students in Kuwait. J Allied Health 2007; 36: $41-46$ (PMID: 17425190).

15. Eldarrat A, Alkhabuli J, Malik A. The prevalence of self-reported halitosis and oral hygiene practices among Libyan students and office workers. Libyan J Med 2008; 3: 170-176 (PMCID: 3074308) (doi: 10.4176/080527).

16. Azodo CC, Ehigiator O, Ehizele AO, Ololo O. Medical doctors' knowledge of dental specialty: Implication for referral. Saratov J Med Sci Res 2010; 6: 140-143.

17. Masalu J, Mtaya M, Astrøm AN. Risk awareness, exposure to oral health information, oral health related beliefs and behaviours among students attending higher learning institutions in Dar es Salaam, Tanzania. East Afr Med J 2002; 79: 328-333 (PMID: 12638824).

18. Carstens IL, Hartshorne JE, De Vos D, Blignaut RJ. Oral health status and treatment needs of black college students at Khayelitsha. J Dent Assoc S Afr 1991; 46: 535-537 (PMID: 1820671).

19. Lukacs JR. Gender differences in oral health in South Asia: Metadata imply multifactorialbiological and cultural causes. Am J Hum Biol 2011. 23: 398-411 (PMID: 21448908) (doi: 10.1002/ajhb.21164).

Authors:

Clement C. Azodo - BDS, MSc Physio, MPH, FMCDS, Department of Periodontics, University of Benin Teaching Hospital, Benin City, Nigeria;

Barnabas Unamatokpa - BDS, Department of Periodontics, University of Benin Teaching Hospital, Benin City, Nigeria. 